\title{
ESTRUCTURA DE LA MACROFAUNA EN AMBIENTES OXICOS DE BAHIA COLIUMO, REGION DEL BIO-BIO, CHILE CENTRAL
}

\author{
STRUCTURE OF MACROFAUNA IN OXIC ENVIRONMENTAL OFF BAY \\ COLIUMO, BIO-BIO REGION, CENTRAL CHILE
}

\author{
Karina Neira ${ }^{1}$, \& Maritza Palma ${ }^{2}$ \\ ${ }^{1}$ Departamento de Oceanografía, Universidad de Concepción, Casilla 160-C, Concepción, Chile. \\ ${ }^{2}$ Centro de Investigación Oceanográfica en el Pacífico Sur-Oriental (COPAS), Departamento de Oceanografía, \\ Universidad de Concepción, Casilla 160-C, Concepción, Chile. kaneira@udec.cl
}

\begin{abstract}
RESUMEN
Se analizó la densidad, biomasa, diversidad y riqueza de especies de la macrofauna bentónica ( $\geq 500 \mu \mathrm{m})$ en ambientes óxicos $\left(>0.5 \mathrm{ml} \mathrm{L}^{-1}\right)$ en Bahía Coliumo $\left(36^{\circ} 32^{`} \mathrm{~S}, 7^{\circ} 57^{\circ} \mathrm{W}\right)$, Chile central. En un transecto de cinco estaciones, entre 3,6 y 22,5 m de profundidad, fueron recolectadas muestras de sedimento con una draga tipo Van veen de $0,051 \mathrm{~m}^{2}$, a bordo de la embarcación científica L/C Kay-Kay. A partir de las muestras de sedimento se determinó, el porcentaje de materia orgánica total, el tamaño de grano, densidad y biomasa de la macrofauna. Adicionalmente, se tomaron muestras de agua para el análisis de oxígeno disuelto de fondo, temperatura y salinidad. Las densidades de la macrofauna total fluctuaron entre 1111 y 11183 ind $\mathrm{m}^{-2}$. En tanto, los valores de biomasa alcanzaron 9,9 y $252,86 \mathrm{~g} \mathrm{~m}^{-2}$. Del total de la macrofauna 29 especies fueron identificadas. Los valores más altos de diversidad $(3,23)$ coinciden con los valores más bajos de dominancia y uniformidad. Los parámetros de diversidad no mostraron una relación lineal con la profundidad. Los poliquetos fueron el grupo dominante $(51,8 \%)$, seguido de los crustáceos $(41 \%)$ y moluscos $(6,8 \%)$. Adicionalmente, a través del análisis BIOENV, se observó que el porcentaje de arena y el contenido de materia orgánica fueron los principales factores ambientales que regulan la estructura de la macrofauna de Bahía Coliumo.
\end{abstract}

Palabras claves: Bentos marino, diversidad, factores ambientales.

\section{ABSTRACT}

The density, biomass, diversity and species richness of macrofaunal benthic $(\geq 500 \mu \mathrm{m})$ in oxic environment $(>0.5$ $\left.\mathrm{ml} \mathrm{L}^{-1}\right)$ off Bay Coliumo $\left(36^{\circ} 32^{`} \mathrm{~S}, 72^{\circ} 57^{`} \mathrm{~W}\right)$, Chile were analyzed. One transect of five stations between 3.6 and $22.5 \mathrm{~m}$ of depth were collected samples of sediments with a dredged Van veen of $0.051 \mathrm{~m}^{2}$ a board R/V Kay-Kay in August (winter) of 2004. From sediment sample the organic matter percent, size of grain, density and biomass of the macrofauna were determined. Additionally, were taken water column samples for analyses of dissolved oxygen of bottom, temperature and salinity. The densities of macrofauna fluctuated between 1,111 and 11,183 ind $\mathrm{m}^{-2}$. While the values of biomass to reached 9.9 and $252.86 \mathrm{~g} \mathrm{~m}^{-2}$. For all macrofauna 29 species were identified. The highest values of diversity (3.23) coincided with lowest values of dominance and evenness. The parameters of diversity did not show a lineal relationship with depth. The polychaetes were dominant group (51.8\%) followed by the crustacean $(41 \%)$ and molluscs $(6.8 \%)$. In addition, through of analysis of BIOENV observed that percent of sand and content of organic matter are the main environment factors that regulate the communities structure of macrofauna off Bay Coliumo.

KEYwords: Marine benthos, diversity, environmental factors. 


\section{INTRODUCCION}

Hasta la fecha no se han realizado estudios de la macrofauna bentónica litoral y sublitoral somera en el área ubicada en las proximidades de bahía Coliumo. A excepción de los registros de Soto (1997), a profundidades similares, cuyos muestreos fueron realizados en otoño. Aquí se indica la presencia de escasos grupos taxonómicos, un bajo número de especies, de altas dominancias y una baja diversidad. En general, diversos estudios realizados en el Pacifico Sur-Oriental se refieren a los patrones de distribución batimétrica de la biota bentónica (Gallardo 1963, Frankenberg \& Menzies 1968, Rosenberg et al. 1983, Arntz et al. 1991). Aunque, en las costas chilenas la mayoría de estas contribuciones han sido desarrolladas en el centro sur de Chile (Carrasco \& Gallardo 1994, Gallardo et al. 1995, Carrasco et al. 1999, Gutiérrez et al. 2000) y en menor grado en el norte de Chile (Gallardo 1963, Carrasco 1997, Quiroga et al. 1999). La mayor parte de estos estudios coinciden en que las comunidades bentónicas sublitorales presentan una baja diversidad, elevadas biomasas, elevadas abundancias, una marcada dominancia numérica de organismos pequeños, dominancia de poliquetos y una elevada biomasa de bacterias filamentosas (Thioploca araucae y $T$. chileae).

La plataforma continental frente a Concepción ha sido caracterizada por presentar centros activos de surgencias (Fonseca \& Farías 1987), de una alta productividad primaria (>1 g C m-2 d-1) (Daneri et al. 2000), y sujeta a la influencia de una zona de mínimo oxígeno que sostiene una de las mayores pesquerías del mundo (Arcos et al. 2001). Estos sistemas además, presentan una fuerte variación estacional que se manifiesta por una intensificación del afloramiento costero durante la época de primavera-verano (Shaffer et al. 1999). Aunque, en invierno ocurre un mayor aporte de agua dulce por los ríos y lluvias, provocando bajas salinidades superficiales, que se extienden considerablemente hacia el océano (Strub et al. 1998). A la variabilidad estacional se agregan fluctuaciones inter-anuales, que se derivan de procesos de gran escala, incluyendo el ciclo ENOS. Aunque en términos generales el ecosistema marino de la región refleja condiciones invernales normales para la región y ausencia de anomalías oceanográficas asociadas al fenómeno El Niño (FIP 2005).
De esta forma y según lo planteado anteriormente, el objetivo principal de este estudio consiste en determinar la densidad, biomasa, diversidad y riqueza de especies de las comunidades de la macrofauna de Bahía Coliumo. Asimismo, investigar los factores ambientales que podrían estar determinando la estructura comunitaria en condiciones de invierno, cuando existe ausencia de condiciones hipóxicas en el área de estudio.

\section{MATERIALES Y METODOS}

\section{Área de estudio}

El área de estudio comprende un transecto en el centro de Bahía Coliumo (Fig.1), Región del BíoBío, Chile central. Bahía Coliumo, se encuentra entre los $36^{\circ} 32^{\prime} \mathrm{S}$ y $72^{\circ} 57^{\prime} \mathrm{W}$, inmediatamente al nordeste de Bahía Concepción. Presenta una orientación norte-sur y su boca está delimitada por Punta Pingueral en la parte oriental y Punta Blanca, en su parte occidental.El área de estudio, presenta profundidades menores de 5 metros. El fondo es de pendiente suave y sin accidentes importantes, alcanzando una mayor profundidad (20-25 metros) cerca de la boca de la Bahía (Sobarzo 1984).

De acuerdo a las características hidrográficas de Bahía Concepción y áreas adyacentes, Ahumada \& Chuecas (1979) señalan, que durante períodos primavera-verano y hasta principios de otoño, la influencia de las aguas ecuatoriales subsuperficiales de la contracorriente Chile-Perú, provenientes de eventos de surgencias, resultan de mayor importancia. Por lo tanto, esta masa de agua se caracteriza por contener bajos niveles de oxígeno $(<1 \mathrm{ml} / \mathrm{L})$, salinidades relativamente altas $(>34,5$ PSU) y temperaturas relativamente bajas $\left(10-12^{\circ} \mathrm{C}\right)$ (Ahumada et al. 1983).

\section{Muestreo BenTóNICO}

Las muestras fueron recolectadas, en cinco estaciones durante el mes de agosto 2004, a lo largo de un transecto desde la boca del estuario en dirección norte de Bahía Coliumo, con un gradiente de profundidad de 3 a 22 metros. Cuatro réplicas de sedimento para cada estación fueron tomadas a bordo de la embarcación científica L/C Kay Kay de la Universidad de Concepción, con una draga tipo Van veen de $0,051 \mathrm{~m}^{2}$. Una réplica considerada para el análisis de granulometría y porcentaje de 
materia orgánica total y tres réplicas para el análisis de la macrofauna.

Las muestras correspondientes al material biológico, fueron preservadas en alcohol al 70\%, previamente fijadas, en una solución de formalina al $10 \%$. Se realizó el lavado y tamizado de las muestras por medio de reflotación del sedimento, a través de un tamiz de $500 \mu \mathrm{m}$. El material fue almacenado en frascos plásticos, debidamente etiquetados para su posterior análisis.

Adicionalmente, se tomaron muestras de agua para el análisis de la concentración de oxígeno disuelto, temperatura y salinidad en cada estación, mediante botellas Niskin con termómetro de inversión.

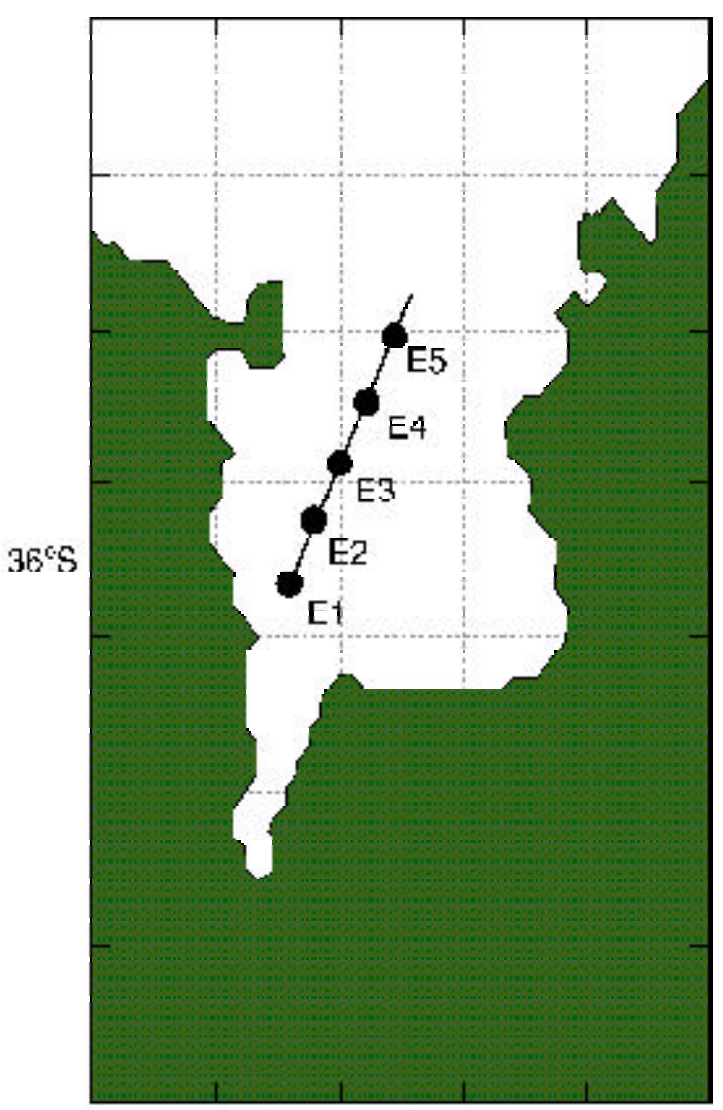

7204

FIGURA 1. Ubicación geográfica de las cinco estaciones analizadas en Bahía Coliumo.

Figure 1. Geographic location of the five stations analyzed in Coliumo Bay.

\section{ANÁLISIS DE MUESTRAS}

ANÁLISIS DE L A MACROFAUnA

La identificación del material biológico se realizó con la ayuda de una lupa estereomicroscopio. Los especimenes fueron analizados hasta al nivel taxonómico más bajo posible. La biomasa fue estimada como peso húmedo, utilizando una balanza analítica electrónica de precisión $0,1 \mathrm{mg}$.

Determinación de $\mathrm{O}_{2}$ Disuelto, temperatura y SALINIDAD

Las muestras de oxígeno fueron analizadas mediante el método de Winkler y microtituladas con un DOSIMAT (Knap et al. 1993) en tres réplicas. La temperatura se registró mediante un termómetro de inversión y la salinidad por medio de un salinómetro Beckman.

ANÁLISIS DE SEDIMENTO

Se determinó el contenido de materia orgánica total para cada estación, el cual fue estimado a través de la pérdida de peso por calcinación de acuerdo a lo propuesto por Postman (1954), mientras que el análisis granulométrico se realizó mediante tamices geológicos (Buchanan \& Kain 1971). Para la clasificación de los sedimentos se utilizó la escala de Udden-Wentworth (Folk 1974).

ANÁLISIS DE DATOS

Los valores promedios de densidad, biomasa y desviación estándar fueron calculados para cada estación y estandarizados a $\mathrm{m}^{2}$. Los cálculos de diversidad y riqueza específica, fueron estimados a partir de los datos agrupados por estación, a través de los siguientes índices:

\section{Shannon-Wiener Hº (Pielou 1966):}

$$
\mathrm{H}^{\prime}=-\Sigma \text { pi lnpi }
$$

Donde $\mathrm{pi}=\mathrm{ni} / \mathrm{N}$ y ni= número de individuos de la iésima especie y $\mathrm{N}=$ número total de individuos.

Cálculo de Equitabilidad o Uniformidad J' (Pielou 1966):

$$
\mathrm{J}^{\prime}=\mathrm{H}^{\prime} / \ln \mathrm{S}
$$

Donde $\mathrm{S}=$ número de especies 
Dominancia de Simpson D (Legendre \& Legendre 1979)

$$
\mathrm{Cs}=\mathrm{S}(\mathrm{ni}(\mathrm{ni}-1)) /(\mathrm{N}(\mathrm{N}-1))
$$

A través de las curvas de rarefacción de SandersHurlbert se evaluó la riqueza de especies corregida por tamaño de muestra (Sanders et al. 1965, Hurlbert 1971).

Los índices de diversidad fueron estimados mediante el software estadístico PRIMER 5.0 (Clarke \& Gorley 2001).

La clasificación numérica fue analizada a través del índice de Bray Curtis como coeficiente de asociación comunitario, con la estrategia jerárquica de la media ponderada (UPGMA) (Sneath \& Sokal 1973). La ordenación comunitaria (Gauch 1982), fue realizada mediante un análisis de escalación No Métrica Multidimensional (NMDS) (Field $e t$ al. 1982), generándose una matriz de asociación con el índice de Bray Curtis, previa transformación de los datos a la forma $Y=\sqrt{ } \sqrt{ }$.

Como una manera de describir la distribución de las dominancias entre la comunidad, se trazaron curvas de k-dominancia (Lambshead et al. 1983). Las correlaciones entre factores ambientales (Contenido de arena (\%), \% Materia orgánica, Oxígeno disuelto, temperatura, salinidad y profundidad) y parámetros comunitarios (densidad, biomasa y parámetros de diversidad) fueron estimados con el coeficiente «r» de Pearsons. Y la relación entre los factores ambientales y parámetros comunitarios fueron estimados por medio del análisis de Bio-Env (Clarke \& Ainsworth 1993). El porcentaje de contribución de las especies dominantes de la macrofauna fue identificada mediante el procedimiento SIMPER (Clarke 1993).

\section{RESULTADOS}

\section{Parámetros Ambientales}

En la Tabla I aparecen los valores de oxígeno disuelto (OD), salinidad (S), temperatura (T), porcentaje de materia orgánica (MOT) y porcentaje de arena (A). Los niveles de OD fluctúan entre 1,93 y 2,49 ml L-1. Los valores más altos de oxígeno fueron registrados en la estación 1 cerca del estuario (2,49 ml L- 1$)$, los cuales disminuyeron hacia la estación 3 (1,94 ml L-1), y luego aumentaron levemente hacia las estaciones más profundas 4 y 5 (2,03 y $1,95 \mathrm{ml} \mathrm{L-1}$ respectivamente). Distribución contraria, presentó el porcentaje de materia orgánica de los sedimentos, registrando los valores más bajos la estación $1(2,5)$, y los más altos la estación 3 $(9,49)$, para luego disminuir nuevamente hacia las estaciones más profundas 4 y 5 (8,95 y 5,22 respectivamente).

Visualmente es posible identificar sedimentos de alto porcentaje de arenas, lo cual coincide con el análisis textural. Sin embargo el porcentajes de fango aumenta con la profundidad.

TAbla I. Datos ambientales de la zona de estudio; Oxígeno disuelto (OD), salinidad (S), temperatura (T), Porcentaje de materia orgánica (MOT) y porcentaje de arena (A).

TABLE I. Environmental data of the zone of study; Dissolved oxygen (OD), salinity (s), temperature (t), Percentage of organic matter (MOT) and percentage of sand (a).

\begin{tabular}{|c|c|c|c|c|c|c|c|c|}
\hline Estación & $\begin{array}{l}\text { Profundidad } \\
\text { (m) }\end{array}$ & \multicolumn{2}{|c|}{$\begin{array}{l}\text { Latitud } \\
\left({ }^{\circ} \mathrm{S}\right)\end{array}$} & $\begin{array}{c}\text { OD } \\
\left(\mathrm{ml} / \mathrm{L}^{1}\right)\end{array}$ & $\begin{array}{c}\mathrm{S} \\
\mathrm{psu}\end{array}$ & $\begin{array}{c}\mathrm{T} \\
\left({ }^{\circ} \mathrm{C}\right) \\
\end{array}$ & $\begin{array}{c}\text { MOT } \\
(\%)\end{array}$ & $\begin{array}{l}\text { A } \\
(\%) \\
\end{array}$ \\
\hline 1 & 3,6 & $36^{\circ} 32^{\prime} 78^{\prime \prime}$ & $72^{\circ} 57^{\prime} 16^{\prime \prime}$ & 2,493 & 28,3 & 13,3 & 2,506 & 95,38 \\
\hline 2 & 8,8 & $36^{\circ} 32^{\prime} 29^{\prime \prime}$ & $72^{\circ} 57^{\prime} 19^{\prime \prime}$ & 2,455 & 33,5 & 12,9 & 3,233 & 98,38 \\
\hline 3 & 12,8 & $36^{\circ} 31^{\prime} 88^{\prime \prime}$ & $72^{\circ} 57^{\prime} 04^{\prime \prime}$ & 1,939 & 34,3 & 12,9 & 9,491 & 74,71 \\
\hline 4 & 19,5 & $36^{\circ} 31^{\prime} 54^{\prime \prime}$ & $72^{\circ} 56^{\prime} 69^{\prime \prime}$ & 2,038 & 34,4 & 12,9 & 8,946 & 86,14 \\
\hline 5 & 22,5 & $36^{\circ} 31^{\prime} 16^{\prime \prime}$ & $72^{\circ} 56^{\prime} 86^{\prime \prime}$ & 1,948 & 34,4 & 12,8 & 5,226 & 78,17 \\
\hline
\end{tabular}


COMPOSICIÓN FAUNística

Al analizar la composición taxonómica de la macrofauna de Bahía Coliumo (Fig. 2), destaca la presencia de cuatro grupos taxonómicos mayores: Annelida (Polychaeta 51,8\%), Crustacea (Amphipoda y Ostracoda, 41\%), Mollusca (Gastropoda y Bivalvia, 6,8\%) y Nemertea $(0,46 \%)$.

La dominancia de poliquetos aumentó hacia la boca de la Bahía, registrando en el área más somera (estación 1) un 25,3 \% y un 91,8\% en la estación 5. Por el contrario, los crustáceos estuvieron mejor representados en las estaciones 1 y 2 , donde alcanzan un 45 y $71 \%$ de dominancia, respectivamente. Finalmente, los moluscos siempre alcanzaron bajos porcentajes de representatividad a lo largo de la transecta, a excepción de la estación 1, donde se observó una contribución del 23\% de dominancia con respecto al total de la macrofauna. En general, la contribución del grupo Polychaeta estuvo compuesta por especies comoMediomastus branchiferus, Nepthys ferruginea, Lumbrineris tetraura, Cossura chilensis y Paraprionospio pinnata con distintas frecuencias de ocurrencia a lo largo de la transecta. Las especies mejor representadas en la estación 1, fueron Mediomastus branchiferus y Tharyx spp. (78, 4 y 26, 14\% respectivamente). En la estación 2 aparecieron además especies como Paraprionospio pinnata, Lumbrineris chilensis, Mediomastus branchiferus y Nepthys ferruginea. Mientras que la estación 3, se encuentran mejor representadas por las especies Paraprionospio pinnata y Cossura chilensis. Finalmente, en las estaciones 4 y 5 Paraprionospio pinnata es la especie dominante, aunque con bajas densidades. Por otra parte, los crustáceos peracáridos como Ampelisca araucana $(35,5 \%)$ fueron los organismos dominantes,. En tanto, el bivalvo Thyasira sp presentó el más alto porcentaje entre los moluscos, contribuyendo con un $4,8 \%$ de dominancia. Finalmente, dentro del grupo miscelánea, fueron registrados organismos del grupo Nemertea, especímenes que sólo contribuyeron con un $0,48 \%$ al total de la macrofauna de Bahía Coliumo.

\section{Abundancia y Biomasa}

Los máximos valores de densidad promedio (Fig.3) de la macrofauna fueron registrados en la estación 3 con un valor promedio de 11.183 ind $\mathrm{m}^{2}$. Mientras los valores más bajos fueron observados en las estaciones 1 y 5 con 1.111 y 5.013 ind $\mathrm{m}^{2}$, respectivamente. La biomasa promedio (Fig.4) aumentó con la profundidad hasta la estación 4 , con 252,86 $\mathrm{g} \mathrm{m}^{-2}$, disminuyendo nuevamente hasta $80,25 \mathrm{~g} \mathrm{~m}^{2}$, en la estación 5 .

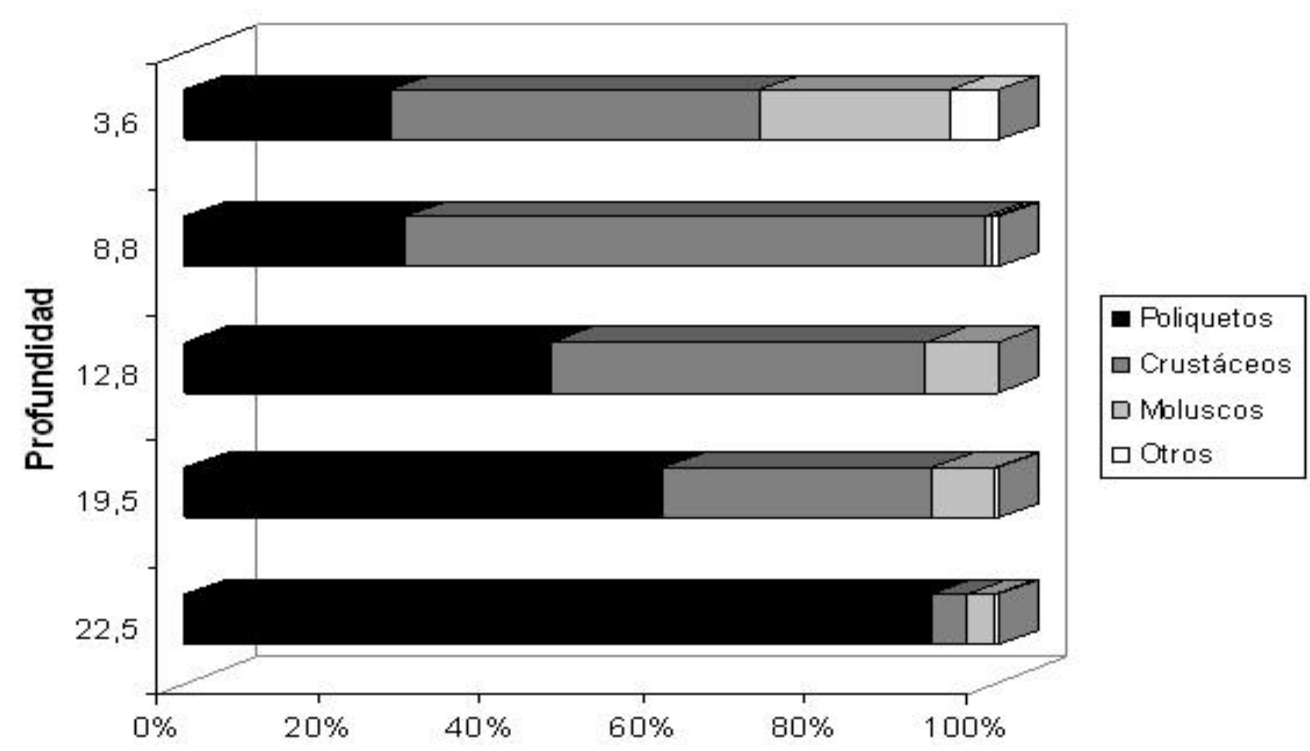

FIgURA 2. Contribución porcentual de los grupos taxonómicos mayores en Bahía Coliumo.

FIGURE 2. Percentage contribution of the greater taxonomic groups in Coliumo Bay. 


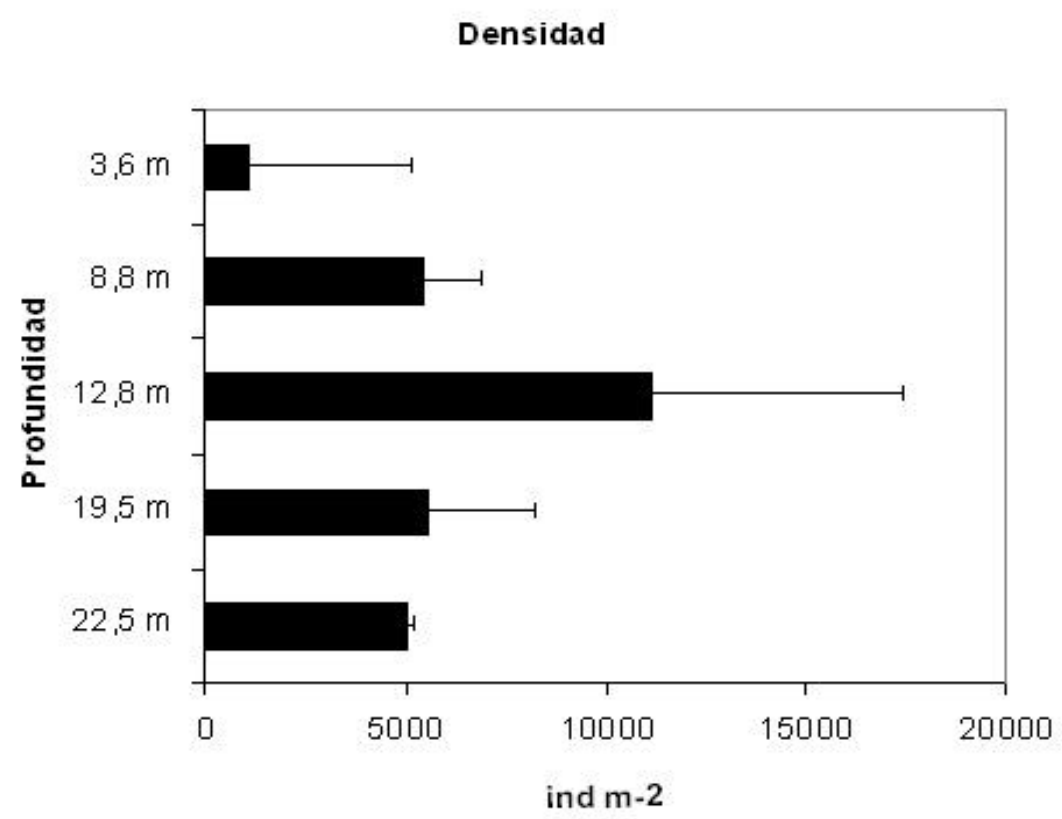

Figura 3. Densidad de la macrofauna en cada estación analizada en Bahía Coliumo.

Figure 3. Density of the macrofauna in each station analyzed in Coliumo Bay.

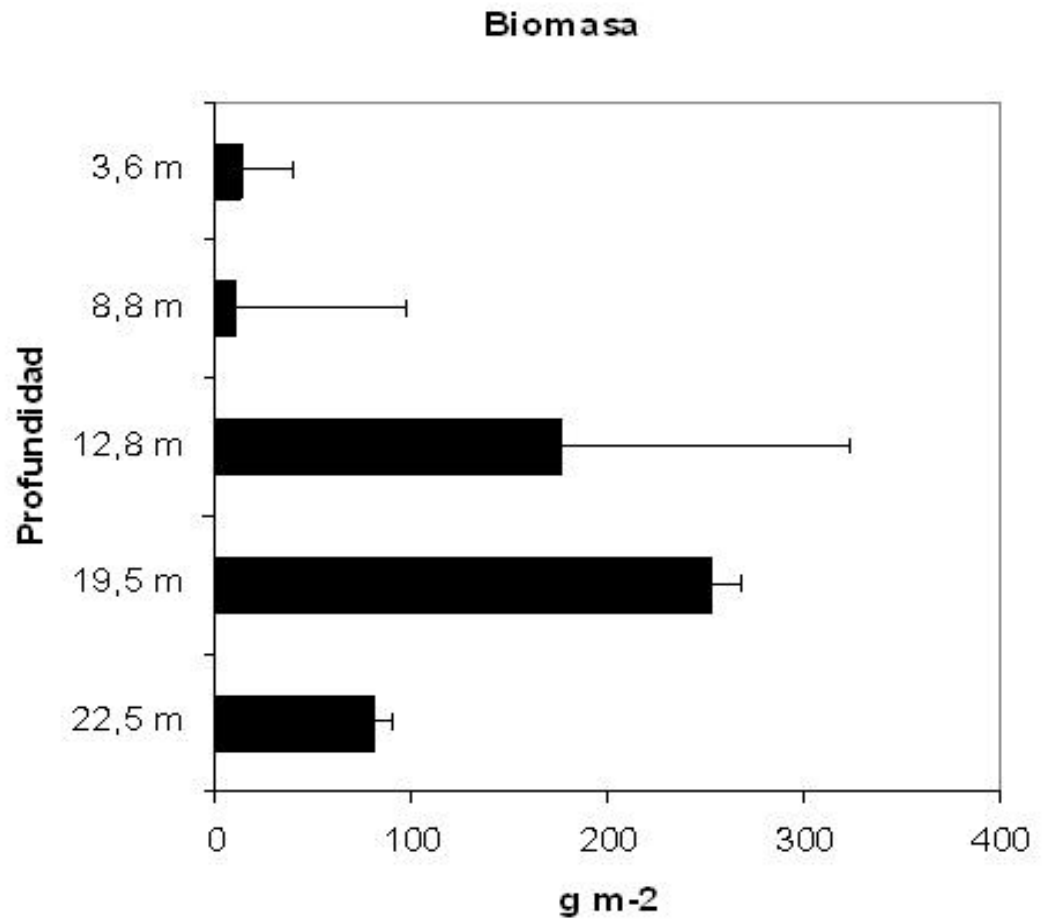

Figura 4. Biomasa de la macrofauna en cada estación analizada en Bahía Coliumo.

FiguRE 4. Biomass of the macrofauna in each station analyzed in Coliumo Bay. 
Diversidad y RiQueZa de EsPECIES

La riqueza de especies fue particularmente baja, donde se identificó un total de 29 especies con un rango de 13 a 20 especies por estación. Distinta situación se observó para el índice de diversidad, el cual asignó valores claramente altos para las todas estaciones, variando entre 3,23 y 2,00. El índice más alto fue observado en la estación $1(3,23)$ y el más bajo en la estación 2 (2,00). En general, se encontró bajos valores de dominancia, presentando la estación 2 los valores más altos de dominancia $(0,45)$ y más bajos de uniformidad (Tabla II).

La Fig.5 muestra las curvas de rarefacción. En general, estas presentaron una baja pendiente en todas las estaciones, situación que indica baja diversidad específica, especialmente la estación 3.
Sin embargo, la estación más someras (1) presentó una curva con mayor pendiente, aunque de corta longitud debido a la baja densidad de especies. No obstante, indica mayor diversidad y coincide así con los resultados entregados por el índice de diversidad de Shannon analizado anteriormente. Por el contrario, las estaciones 2 y 3 se diferenciaron con una menor diversidad, reafirmando también la menor diversidad signada por el índice.

La Figura 6 muestra las curvas del porcentaje de dominancia acumulada para cada una de las estaciones. Aquí claramente se observa una mayor dominancia ecológica en la estación 2, la cual coincide con los valores más bajos de diversidad. En tanto, los porcentajes de dominancia más bajos fueron observados en las estaciones 1 y 4 .

TABLA II. Valores calculados en forma agrupada para cada estación, índices de diversidad en Bahía Coliumo: número de especies $(\mathrm{S})$, diversidad específica de Shannon $\left(\mathrm{H}^{\star}\right)$, Rarefacción $(\mathrm{ES}(51))$, diversidad máxima teórica $\left(\mathrm{H}^{\text {‘max }}\right)$, uniformidad o equidad (J'), e índice de dominancia de Simpson (D).

TABLE II. Values calculated in form grouped for each station, indices of diversity in Coliumo Bay: number of species (s), specific diversity of Shannon (H'), Rarefacción (IT IS (51)), theoretical Maxima diversity (H 'max), uniformity or fairness ( $\mathrm{J}$ '), and index of dominance of Simpson (d).

\begin{tabular}{ccccccc}
\hline Estación & $\mathrm{S}$ & $\mathrm{H}^{`}\left(\log _{2}\right)$ & $\mathrm{ES}(51)$ & $\mathrm{H}^{`} \max$ & $\mathrm{J}^{`}$ & $\mathrm{D}$ \\
\hline 1 & 15 & 3,23 & 11,93 & 3,91 & 0,83 & 0,13 \\
2 & 19 & 2,00 & 8,48 & 4,25 & 0,47 & 0,45 \\
3 & 13 & 2,14 & 7,12 & 3,70 & 0,58 & 0,31 \\
4 & 20 & 2,83 & 9,97 & 4,32 & 0,65 & 0,20 \\
5 & 17 & 2,93 & 10,43 & 4,09 & 0,72 & 0,19 \\
\hline
\end{tabular}

Clasificación y ORdenación

Los análisis de clasificación y ordenación (Fig. 7 y 8) indican la formación de tres grupos principales: grupo I, II y III. El grupo I, definido por las asociaciones faunísticas entre las réplicas de la estación 1. El grupo II por la asociación de las réplicas de la estación 3 y el grupo III definido por las réplicas de las estaciones 2,4 y 5.

De acuerdo al análisis SIMPER (Tabla III), Thyasira sp. Es la especie que contribuye en un alto porcentaje al grupo I con un $38.85 \%$ de dominancia. En tanto, A. araucana destaca en el grupo II y III con un 31,03 y $37,58 \%$ de dominancia, respectivamente.
TABLA III. Porcentaje de contribución de las especies para cada grupo obtenido por Análisis de clasificación numérica

TABLE III. Percentage of contribution of the species for each group obtained by Analysis of numerical classification

\begin{tabular}{ccc}
\hline Grupo & Especie & $\%$ \\
\hline I & Thyasira sp. & 38,85 \\
II & Ampelisca araucana & 31,03 \\
III & Ampelisca araucana & 37,58 \\
\hline
\end{tabular}


RELACIÓN ENTRE PARÁMETROS BIOLÓGICOSY AMBIENTALES De acuerdo al análisis de correlación de Pearsons (Tabla IV), se encontró que el porcentaje de arena se correlaciona negativamente con la abundancia de poliquetos $(\mathrm{r}=-0,70)$. En cambio, la densidad $(\mathrm{r}=0,78)$ y biomasa $(\mathrm{r}=0,95)$ mostró una positiva correlación con el porcentaje de MOT $(\mathrm{p}<0,001)$. En tanto, el oxígeno disuelto se correlacionó negativamente con la biomasa $(\mathrm{r}=-0,75)$ y los poliquetos $(\mathrm{r}=-0,79)$. La salinidad mostró una negativa correlación con los moluscos $(r=-0,88)$, pero a su vez una positiva correlación con la temperatura $(r=0,95)$. Los poliquetos estuvieron positiva y significativamente relacionados con la profundidad ( $\mathrm{r}=0,93 ; \mathrm{p}<0,05)$.

En tanto, el análisis BIOENV indicó que las mejores correlaciones $(\rho \mathrm{w}=1,00)$ estuvieron relacionadas entre el porcentaje de arena, contenido de materia orgánica y las matrices de abundancia de la macrofauna de Bahía Coliumo.

Tabla IV. Valores obtenidos de acuerdo al análisis de correlación de Pearsons para la zona estudiada.

TABLE IV. Values obtained according to the analysis of correlation of Pearsons for the studied zone.

\begin{tabular}{lrrrrrr}
\hline & $\%$ A & MOT & OD & S & T & Z \\
\hline S & $0,44^{\text {o }}$ & $-0,10$ & 0,14 & 0,31 & $-0,45$ & 0,35 \\
$\mathrm{H}^{`}$ & 0,05 & $-0,25$ & 0,06 & $-0,55$ & 0,44 & 0,08 \\
ES(51) & 0,39 & $-0,56$ & 0,38 & $-0,67$ & 0,49 & $-0,11$ \\
H‘max & 0,46 & $-0,15$ & 0,17 & 0,28 & $-0,44$ & 0,34 \\
J & $-0,05$ & $-0,24$ & 0,05 & $-0,64$ & 0,57 & $-0,06$ \\
D & 0,22 & $-0,01$ & 0,18 & 0,44 & $-0,41$ & $-0,16$ \\
Densidad & $-0,69$ & 0,78 & $-0,67$ & 0,72 & $-0,50$ & 0,32 \\
Biomasa & $-0,60$ & 0,95 & $-0,75$ & 0,58 & $-0,37$ & 0,60 \\
Polychaeta & $-0,70$ & 0,36 & $-0,79$ & 0,58 & $-0,68$ & 0,93 \\
Crustacea & 0,64 & $-0,25$ & 0,67 & $-0,24$ & 0,32 & $-0,75$ \\
Mollusca & 0,23 & $-0,25$ & 0,39 & $-0,88$ & 0,95 & $-0,60$ \\
\hline
\end{tabular}

\section{Rarefacción}

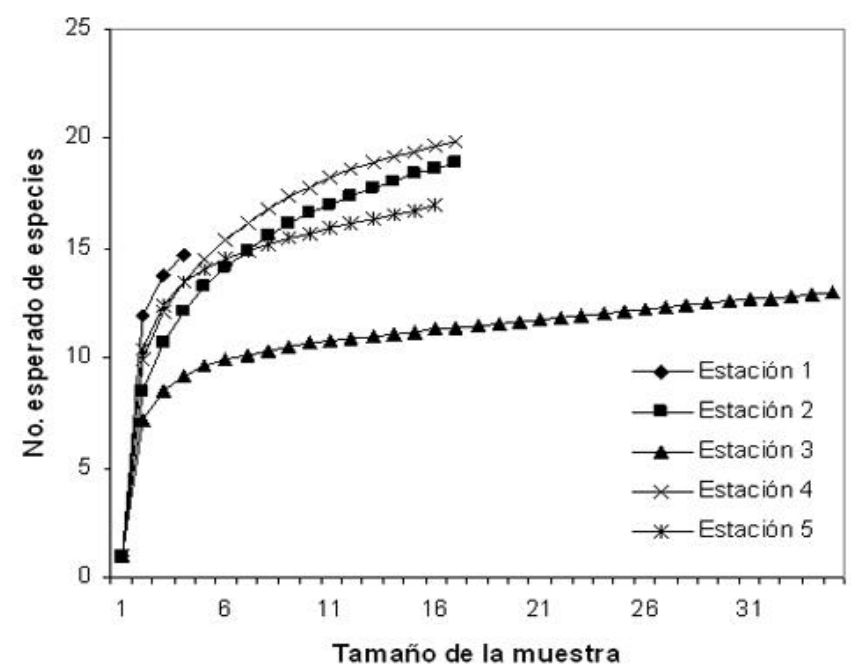

Figura 5. Curvas de rarefacción para las estaciones analizadas.

FiguRE 5. Rarefaction curves for the analyzed stations. 


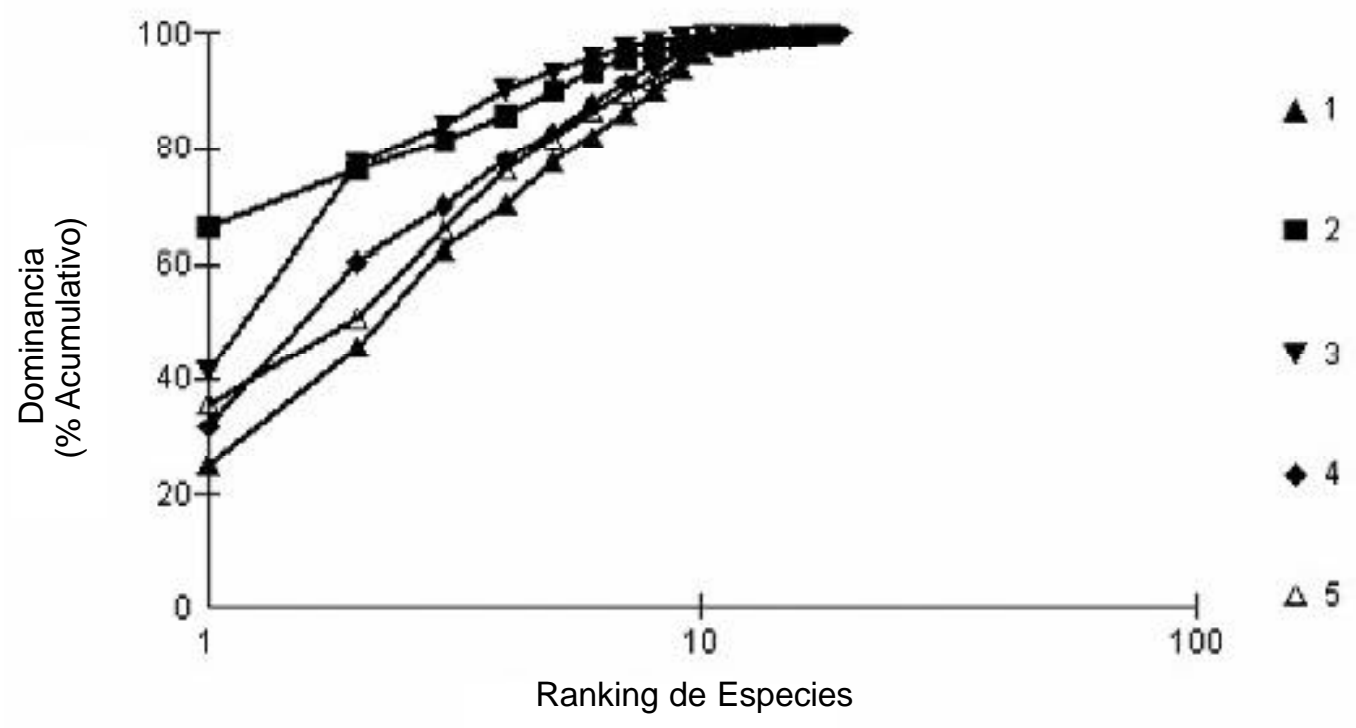

Figura 6.- Curvas de k-dominancia para las comunidades bentónicas analizadas en Bahía Coliumo.

FIGURE 6. Curves of k-dominancia for the analyzed benthics communities in Coliumo Bay.

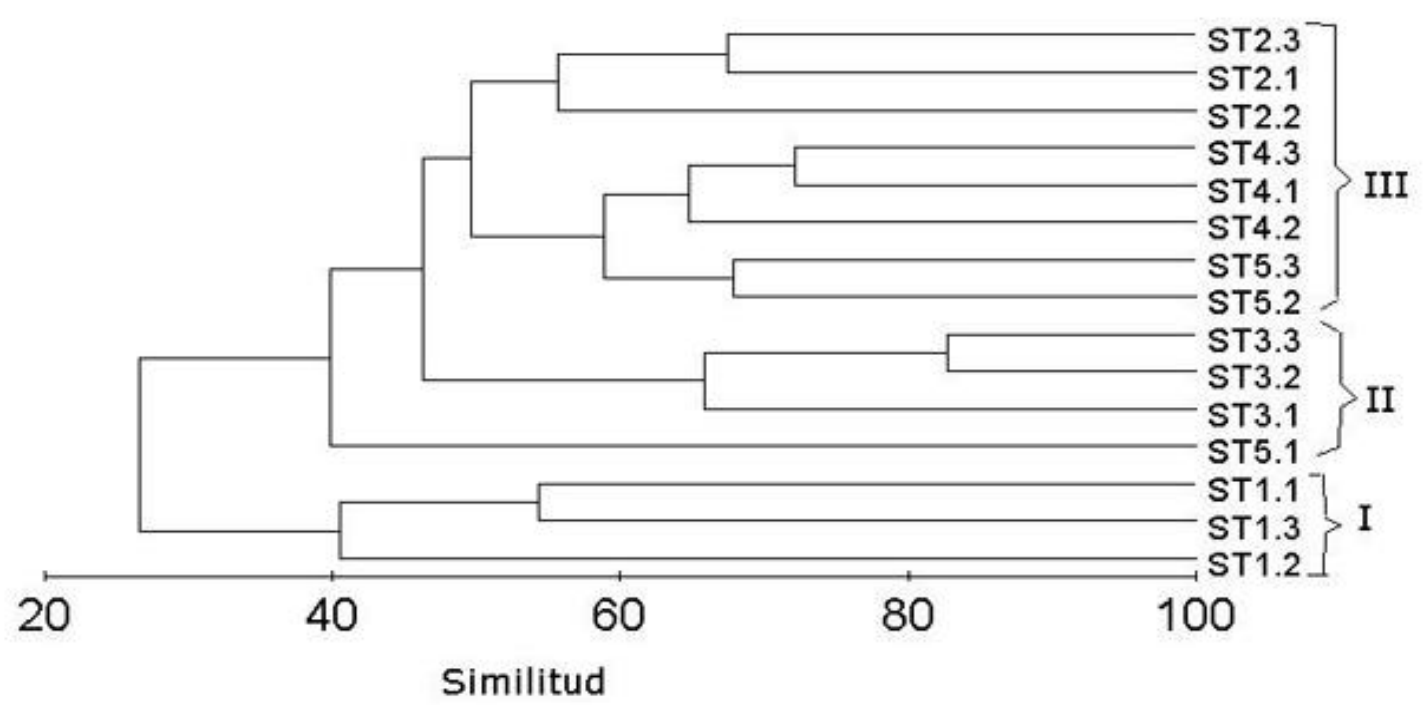

Figura 7. Dendrograma de Clasificación para las réplicas de cada una de las estaciones analizadas en Bahía Coliumo.

FIGURE 7. Dendrogram of classification for the replicate of each one of the stations analyzed in Coliumo Bay. 


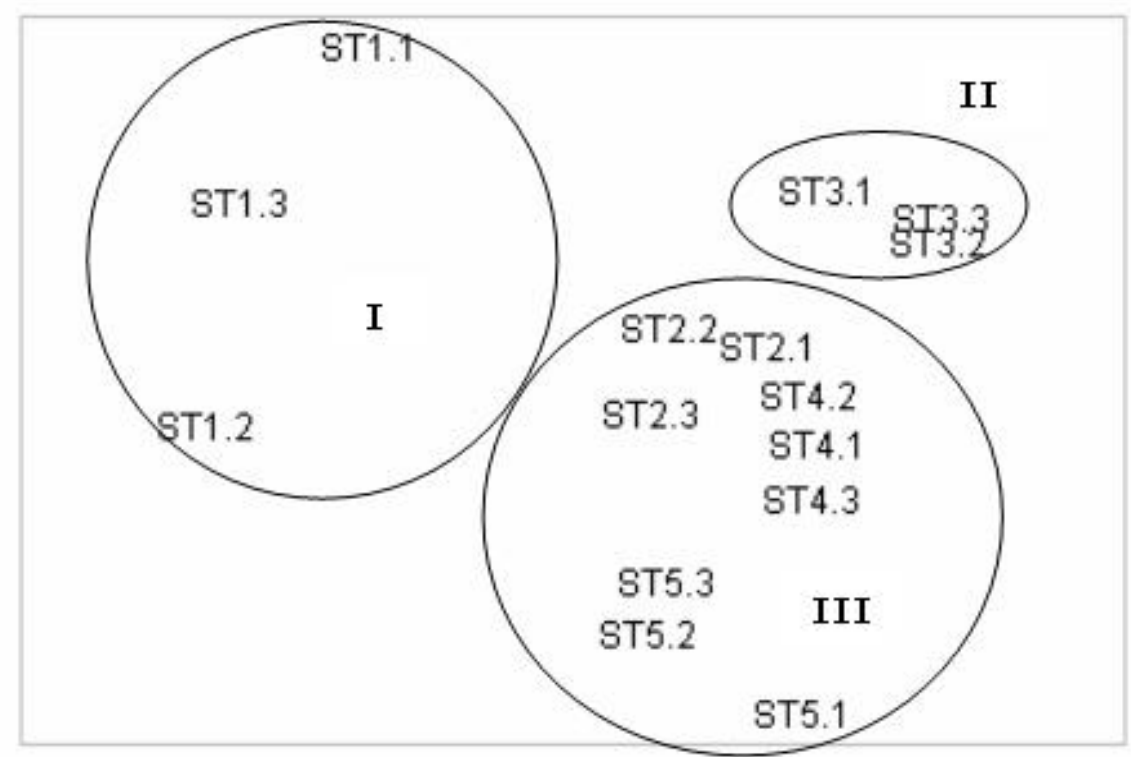

FiguRa 8. Resultados de la ordenación comunitaria para la macrofauna bentónica de Bahía Coliumo.

FIGURE 8. Results of the communitarian arrangement for the macrobenthos of Coliumo Bay.

\section{DISCUSION}

Bahía Coliumo, fue caracterizado por la presencia de sedimentos pobremente seleccionados y un alto porcentaje de arena, el cual disminuye hacia la boca de la Bahía. Los valores relativamente altos de MOT, son consistentes con la alta productividad biológica de la región, indicada previamente por Gutierrez et al (2000) y Farias (2003). Una de las características del sistema de Corrientes de Humboldt es la presencia de una extensiva masa de agua ecuatorial subsupeficial de bajo contenido de oxígeno $(<1 \mathrm{ml}$ $\mathrm{O}_{2} \mathrm{l}^{-1}$ ). En este sentido, los bajos niveles de oxígeno de la plataforma continental del centro sur de Chile, se distribuyen estacionalmente (Ahumada \& Chuecas 1979), siendo bajos en primavera y desapareciendo a comienzos del otoño (Ahumada et al 1983). Según Arcos et al. (1987), el efecto de estos eventos puede alcanzar áreas muy cercanas a la costa $(\mathrm{ca} .4 \mathrm{~km})$, pudiendo ser detectadas incluso dentro de bahías de la zona, como Bahía Concepción y Bahía Coliumo. Aunque, dado lo puntual del muestreo, no podemos corroborar efectos estacionales. Por otra parte, no se encontró diferencias significativas en cuanto a las características físico-químicas del sedimento a través de la transecta.

Adicionalmente, los valores de densidad y biomasa son altos comparados a los resultados de Soto (1997) y a estudios realizados en la plataforma continental frente a Concepción (Gallardo et al 1995; Sellanes et al 2007). Los sedimentos del área más somera, estuvo constituida principalmente de arenas y de bajo contenido de materia orgánica (ca. 3\%), reflejando un moderado régimen de energía. Estos sedimentos estuvieron caracterizados por la presencia de las más bajas abundancias. Mientras la zona media de la transecta (estación 3), presentó los más altos porcentajes de materia orgánica (ca. 10\%), registrando asimismo los más altos valores de densidad de la macrofauna. El límite superior cercano a la boca de la Bahía (estación 5), registró un moderado contenido de materia orgánica (ca. $5 \%$ ), y cuyo conjunto faunístico mostró densidades más bien característico de zonas afectadas periódicamente por condiciones de mínimas de oxígeno. 
Los valores de biomasa fueron altos en comparación a otros estudios realizados en la zona (e.g. Gallardo et al. 1995; Carrasco \& Gallardo 1989; Fossing et al. 1995, Sellanes 2007). Al respecto, estudios previos realizados en la plataforma continental frente a Concepción, han evidenciado que las comunidades bentónicas sublitorales presentan generalmente comunidades de elevadas abundancias y biomasas, una marcada dominancia numérica principalmente de poliquetos de pequeño tamaño, y además una elevada biomasa de bacterias filamentosas (Thioploca araucae y T. chileae). Por lo que, la estructura comunitaria de Bahía Coliumo, coincide en gran parte con resultados obtenidos de otras investigaciones realizadas en la región.

De la misma forma, el número de especies aquí registrado es comparable a otros estudios realizados en la zona durante periodos de invierno (Gallardo et al. 1995, Valdovinos 1998, Sellanes et al. 2007), y bajos valores comparados con la zona norte (Carrasco \& Moreno 2006). Marcadas diferencias fueron observadas en la composición del conjunto faunístico a través de la transecta. Es decir, los crustáceos mejor representados a profundidades someras, reflejan su alta sensibilidad a los niveles de oxígeno (Díaz \& Rosenberg 1995). En tanto, la dominancia de poliquetos aumenta con la profundidad, a medida que los sedimentos se hacen más finos y la heterogeneidad del sedimento disminuye (Levin et al. 2001). En este sentido, Díaz \& Rosenberg (1995) señalan que los poliquetos, representan uno de los taxa más tolerante a bajas concentraciones de oxígeno, seguido por bivalvos y crustáceos.

Tres especies fueron las más dominantes a través de la transecta: Ampelisca araucana, Cossura chilensis y Parapronospio pinnata. Esta última especie, $P$. pinnata alcanzó un $14,5 \%$ de dominancia y se encuentra totalmente ausente en la estación 1. Contrariamente, Soto (1997) encontró un alto grado de dominancia de $P$. pinnata durante el período de muestreo (otoño), alcanzando un $41,4 \%$ de dominancia y un $100 \%$ de ocurrencia. Carrasco (1976) y Carrasco \& Arcos (1980), por su parte indican que las disminuciones invernales en las densidades de $P$. pinnata son consistentes con la historia natural de la especie y su distribución en los sedimentos (Carrasco \& Gallardo 1995).

Los análisis de clasificación numérica y ordenación coinciden con el análisis BIOENV, dado que la formación de grupos faunísticos se encuentran determinados principalmente por las características granulométricas y el contenido de materia orgánica en los sedimentos. Los sedimentos de la estación 1, caracterizados por un ambiente de alta energía, altos niveles de oxígeno disuelto, alto porcentaje de arena, y bajo contenidos de materia orgánica, los cuales coinciden con altos valores de diversidad del conjunto faunístico. Por otro lado los sedimentos de la estación 3, revelan situaciones similares a los encontrados en ambientes de alta carga de materia orgánica, con altas dominancia de algunas especies y baja diversidad (Carrasco \& Gallardo 1995, Gallardo et al. 2004, Valdovinos 1998).

Por lo tanto, a través del análisis BIO-ENV es posible observar que las diferencias en la composición faunística de Bahía Coliumo, al igual que los estudios de Carrasco \& Gallardo (1983), podrían estar reguladas por las características granulométricas y el contenido materia orgánica del sedimento.

\section{AGRADECIMIENTOS}

Agradecemos a la tripulación de la embarcación científica Kay-Kay y especialmente a Luis Aburto, por su participación en la obtención de muestras. Nuestros agradecimientos a Eduardo Quiroga y Javier Sellanes por su cooperación en el manuscrito. Pamela González y Ramiro Riquelme por su ayuda en el análisis de laboratorio, realizados en la estación de biología marina (Dichato) de la Universidad de Concepción. Este trabajo fue financiado por el centro COPAS (FONDAP, CONICYT).

\section{BIBLIOGRAFIA}

Ahumada, R. \& L. Chuecas.1979. Algunas características hidrográficas de la bahía de Concepción $\left(36^{\circ} 40^{\circ} \mathrm{S} ; 73^{\circ} 02^{\prime} \mathrm{W}\right)$ y áreas adyacentes, Chile. Gayana Miscelánea 8: 1-56.

Ahumada, R.,A. \& V. M artínez. 1983. Circulation and fertility of waters in Concepción bay. Estuarine Coastal and Shelf Science. 16: 95-105.

Arcos, D., S. Núñez, L. Castro \& N. Navarro. 1987. Variabilidad vertical de clorofila $a$ en un área de surgencia frente a Chile central. Invest. Pesq., Chile, 34: 47-55.

Arcos, D., L.A. Cubillos \& S.P. NuÑez. 2001. The jack mackerel fishery and El Niño 1997-98 effects off Chile. Progress in Oceanography 49: 597. 617. 
Arntz, W.E., J. Tarazona, V.A. Gallardo, L.A. Flores \& H. SALZWEDEL. 1991. Benthos communities in oxygen deficient shelf and upper slope areas of the Peruvian and Chilean Pacific COSAT, and changes caused by El Niño. In: Modern and Ancient Continental Shelf Anoxia (Eds. Tyson, R. V. \& T. H. Pearson) pp. 131-154 Geological Society Special Publication, London.

BRAY, J.R. \& J. CURTIS. 1957. An Ordination of the Upland Forest Communities of Southern Wisconsin. Ecological Monographs. 27: 325-349.

Buchanan, J.B. \& J.M. Kain. 1971. Measurement of the physical and chemical environment. In: Methods for the Study of marine Benthos (Eds. Holme N.A. \& A.D. McIntyre,), pp.30-58. I.B.P. Handbook No.16. Oxford and Edinburgh: Blackwell Scientific Publication.

Carrasco, F.D. 1976. Larvas de la Familia Spionidae (Polychaeta) en el plancton de la Bahía de Concepción, Chile. Gayana (Zoológica) 38: 163.

Carrasco, F.D. \& D.F. Arcos. 1980. Estimación de la producción secundaria de Paraprionospio pinnata (Spionidae, Polychaeta) frente a Bahía de Concepción, Chile. Boletim do Instituto Oceanografico, Sao Paulo 29: 245-248.

Carrasco, F.D. \& V.A. Gallardo. 1983. Abundance and distribution of the macrobenthic infauna of the Gulf of Arauco, Chile. Internationale Revue der Gesamten Hydrobiologie. 68: 825-838.

Carrasco, F.D. y V. Gallardo. 1989. La contaminación marina y el valor de la macroinfauna bentónica en su evaluación y vigilancia: casos de estudio en el litoral de Concepción, Chile. Biología Pesquera, 18: 15-27.

Carrasco, F.D. \& V.A. Gallardo. 1994. Diversidad, distribución y abundancia del macrobentos del sublitoral y observaciones sobre la dinámica temporal de corto término de los sedimentos de la Bahía de Concepción, Chile. Gayana Oceanológica 2(2): 49-68.

CARrasco, F. D. 1997. The sublittoral macrobenthic fauna off Punta Coloso, Antofagasta, Northern Chile: high persistence of the polychaete assemblage. Bulletin of Marine Science 60(2): 443-459.

Carrasco, F. D., V. A. Gallardo \& M. Baltazar. 1999. The structure of the benthic macrofauna collected across a transect at the central Chile shelf and relationships with giant sulfur bacteria Thioploca spp. Mats. Cahiers de Biologie Marine 40: 195202.

Carrasco, F. D. \& R. A. Moreno. 2006. Long-term dynamics (1990 to 2004) of the polychaete fauna from the sublittoral soft-bottoms off Punta Coloso (Antofagasta), northern Chile. Scientia Marina 70S3 : 169-178.

Clarke, K.R. 1993. Non-parametric multivariate analyses of changes in community structure. Australian Journal of Ecology, 18: 117-143.
Clarke, K.R. \& M. Ainsworth. 1993. A method of linking multivariate community structure to environmental variables. Marine Ecology Progress Series 92: 205-219.

Clarke, K.R. \& R.N. Gorley. 2001. PRIMER v5: User Manual/Tutorial. PRIMER-E: Plymouth, $91 \mathrm{pp.}$

Daneri, G., V. Dellarosa, R. Quiñones, B. Jacob, P. Montero \& O. Ulloa. 2000. Primary production and community respiration in the Humboldt Current System off Chile and associated oceanic areas. Mar. Eco. Prog. Ser., 197:41-49.

Diaz, R.J. \& R. Rosenberg. 1995. Marine benthic hypoxia: a review of its ecological effects and behavioral responses of benthic macrofauna. Oceanography Marine Biology Annual Review 33: 245-303.

FARIAS, L. 2003 Remineralization and accumulation of organic carbon and nitrogen in marine sediments of eutrophic bays: the case of the Bay of Concepción, Chile. Estuarine, Coastal and Shelf Science 57. 829-841.

Field, J.G., K.R. Clarke \& R.M. Warwick. 1982. A practical strategy for analysing multispecies distribution patterns. Marine Ecology Progress Series 8: 37-52.

FolK, R.L. 1974. Petrology of sedimentary rocks. Hemphill Publishing Co., Austin Texas, 159 pp.

Fonseca, T. \& M. Farías. 1987. Estudio del proceso de surgencia en la costa chilena utilizando percepción remota. Investigaciones Pesqueras 34:33-46.

Fossing H, VA G allardo, BB Jørgensen, M Hüttel, LP Nielsen, H Schulz, DE CAnfield, S Forster, RN Glud, JK Gundersen, J Küver, NB Ramsing, A Teske, B Thamdrup \& O Ulloa. 1995. Concentration and transport of nitrate by the mat-forming sulphur bacterium Thioploca. Nature 374: 713-715.

Frankenberg, D. \& R.J. Menzies. 1968. Some quantitative analysis of deep sea benthos off Perú. Deep-Sea Research 15: 623-626.

Gallardo, V.A. 1963. Notas sobre la diversidad de la fauna bentónica sublitoral del norte de Chile. Gayana (Zoológica) 10: 3-15.

Gallardo, V.A. 1985. Efectos del fenómeno de El Niño sobre el bentos sublitoral frente a Concepción, Chile. In: El Niño: su impacto en la fauna marina. (Arntz, W., A. Landa \& J. Tarazona. Eds.), pp.7985. Boletín Instituto del Mar del Perú (vol. extr.).

Gallardo, V.A., F.D. Carrasco, R. Roa \& J.I. Cañete. 1995. Ecological patterns in the benthic macrobiota accros the continental shelf off central Chile. Ophelia 40(3): 167-188.

Gallardo, V.A., M. Palma, F.D. Carrasco, D. Gutiérrez, A. Levin \& J.I. Cañete. 2004. Macrobenthic zonation caused by the oxygen minimum zone on the shelf and slope off central Chile. Deep Sea Research II 51(20-21), 24752490. 
GAUCH, HG. 1982. Multivariate Analysis in Community Ecology. Cambridge University Press, Cambridge, $298 \mathrm{pp}$

Gutierrez, D., V.A. Gallardo, S. Mayor, C. Neira, C. Vásquez, J. Sellanes, M. Rivas, A. Soto, F. Carrasco \& M. Baltazar. 2000. Effects of dissolved oxygen and fresh organic matter on the bioturbation potential of macrofauna in sublittoral bottoms off Central Chile, during the 1997-98 El Niño. Marine Ecology Progress Series 202: 81-99.

Hurlber T, S.H. 1971. The nonconcept of species diversity: a critique and alternative parameters. Ecology 52: 577-586.

Knap, A., A. Michaels, R. Dow, R. Johnson, K. Gundersen, J. Sorensen, A. Close, F. Howse, M. Hammer, N. Bates, A. Doyle \& T. Waterhouse. 1993. Bermuda Atlantic Times-series Studies Methods Manual (Version3). Bermuda biological station for research, Inc., US JGOFS, 108 pp.

Lambshead, P.J.D., H.M. Platt \& K.M. Shaw. 1983. The detection of differences among assemblages of marine benthic species based on an assessment of dominance and diversity. Journal of Natural History. 17: 859-874.

Legendre, L. \& P.Legendre. 1979. Ecologie Numerique. Vol 2. La estructure des donnees ecologiques. Masson, Paris \& Presses de 1'Université du Québec. $254 \mathrm{pp}$.

Levin, L.A., R.J. Etter, M.A. Rex, A.J. Gooday, C.R. Smith, J. Pineda, C.T. Stuart, R.R. Hessler \& D. PAwson. 2001. Environmental influences on regional deep-sea species diversity. Annual Review Ecology and Systematics 132: 51-93.

Palma, M., E. Quiroga, V.A. Gallardo, W. Arntz, D. Gerdes, W. Schneider \& D. Hebbeln. 2005. Macrobenthic animal assemblages of the continental margin off Chile $\left(22^{\circ}\right.$ to $\left.42^{\circ} \mathrm{S}\right)$. Journal of the Marine Biological Association of the United Kingdom 85(2), 233-245.

Pielou, E.C. 1966. The measurement of diversity in different types of biological collections. Journal of Theorical Biology. 13: 131-144.

Postman, A. 1954. Hydrography of the Dutchwadden Sea. Archves Neederlans Zoology. 10:405-511.

Quiroga, E., R. Soto \& N. Rozbaczylo. 1999. Los poliquetos espionidos (Polychaeta: Spionidae) y su importancia en la estructura de una comunidad: un caso de estudio en Bahía Iquique, norte de Chile (20 $\left.11^{\circ} \mathrm{S} ; 70^{\circ} 10^{`} \mathrm{~W}\right)$. Gayana 63 (1): 1-16. Rosenberg, R., W. A rntz, E. Chum, L.A. Flores, G Carbajal, I. Finger \& J. Tarazona. 1983. Benthos biomass and oxygen deficiency in the upwelling system off Peru. Journal Marine Research 41: 263-279.

SAnders, H.L. 1958. Benthic Studies in Buzzards Bay. I. Animal-sediment relationships. Limnology and Oceanography 3 (3): 245-258.

SANDERS, H.L., R.R. HESSLER \& G.R. Hampson. 1965. An introduction to the study of deep-sea benthic faunal assemblages along the Gay Head. Bermuda Transect. Deep-Sea Research 12:845867.

Sellanes, J., E. Quiroga, C. Neira \& D. Gutierrez. 2007. Changes of macrobenthos composition under different ENSO cycle conditions on the continental shelf off central Chile. Continental Shelf Res. 27, 1002-1016.

Shaffer,G., S. Hormazabal, G. Pizarro \& S. Salinas. 1999. Seasonal and interanual variability of currents and temperature off central Chile. J.Geophys.Res. 104: 29951-29961.

Sneath P.H.A. \& R.R Sokal. 1973. Numerical taxonomy. W.H. Freeman. C.A San Francisco.

Sobarzo, M. 1984. Patrón general de circulación (en condiciones invernales) de la bahía de Coliumo $\left(36^{\circ} 32^{`} \mathrm{~S} ; 72^{\circ} 57^{\prime} \mathrm{W}\right)$. Tesis de grado. Universidad de Concepción. Concepción, Chile

Soто, A. 1997. Estudio comunitario de la macroinfauna del sublitoral de la Bahía de Coliumo (36²32'S; $72^{\circ} 57^{\prime} \mathrm{W}$ ), frente a la localidad de Dichato, durante la estación de otoño de 1996. Tesis de grado. Universidad de Concepción. Concepción, Chile.

Strub, P.T., J. Mesias, V. Montecino \& J. Rutland. 1998. Coastal ocean circulation off western South America. In: Robinson, A. Brink, K. (Eds.), The Sea. The global coastal ocean. First workshop on Coastal Ocean Advances Science and Technology Studies (COASTS) IOC, Liege., Vol 2. Wiley, New York.

Valdovinos, C. R. 1998. Patrones de distribución geográfica de la macrofauna bentónica sublitoral en el Golfo de Arauco (Chile Central). Tesis Doctorado, Univ. de Concepción, Chile. 
Macrobentos de bahía Coliumo: NeIRA, K. \& M. PALma

Anexo I. Listado de especies encontradas en Bahía Coliumo y densidad (ind $\mathrm{m}^{-2}$ ) por estación $(\mathrm{n}=3)$.

Annexed I. Listing of species found in Coliumo Bay and density (ind $\left.m^{-2}\right)$ by station $(n=3)$.

\begin{tabular}{lrrrrr}
\hline Especie & St1 & St2 & St3 & St4 & St5 \\
\hline Aricidea & 0 & 4614 & 0 & 20 & 536 \\
Capitellidae & 46 & 0 & 0 & 0 & 0 \\
Cirratulidae & 26 & 6920 & 0 & 0 & 0 \\
Polyonidae & 0 & 384 & 0 & 0 & 7 \\
Sabellidae & 0 & 0 & 0 & 7 & 0 \\
Sigalionidae & 0 & 0 & 0 & 0 & 7 \\
Aphroditidae & 0 & 0 & 0 & 7 & 0 \\
Mediomastus branchiferus s & 78 & 10765 & 59 & 529 & 745 \\
Lumbrineris tetraura & 0 & 16148 & 693 & 118 & 261 \\
Parapronospio pinnata & 0 & 30757 & 46 & 1752 & 1778 \\
Cossura chilensis & 0 & 0 & 3980 & 268 & 0 \\
Pectinaria chilensis & 0 & 0 & 7 & 33 & 163 \\
Isolda viridis & 0 & 0 & 7 & 33 & 242 \\
Nephtys ferruginea & 46 & 13456 & 281 & 438 & 752 \\
Glycera sp & 7 & 769 & 7 & 46 & 33 \\
Spiophanes sp & 78 & 1153 & 0 & 0 & 72 \\
Nereis dorsolobata & 0 & 0 & 0 & 20 & 7 \\
Ampelisca araucana & 176 & 207612 & 4601 & 1595 & 111 \\
Phoxocephalidae 1 & 39 & 1538 & 0 & 0 & 0 \\
Phoxocephalidae 2 & 216 & 14225 & 0 & 0 & 0 \\
Pinnixa valdiviensis & 7 & 1538 & 190 & 20 & 0 \\
Pilumnoides perlatus & 0 & 0 & 0 & 7 & 0 \\
Pseudocorystes sicarius & 20 & 384 & 0 & 0 & 0 \\
Cumaceo & 7 & 0 & 0 & 0 & 0 \\
Ostracoda & 39 & 384 & 333 & 216 & 111 \\
Thyasira sp & 261 & 769 & 765 & 268 & 52 \\
Tagelus dombeii & 0 & 384 & 137 & 13 & 0 \\
Nassarius sp & 0 & 1922 & 78 & 157 & 118 \\
Nemertino & 65 & 1922 & 0 & 13 & 20 \\
\hline & & & & & \\
\hline
\end{tabular}

Recibido: 15.05 .06

Aceptado: 25.07.07 